\title{
ACTUALIZACIÓN EN TORNO A LA EMOCIÓN EXPRESADA: CONSIDERACIONES TEÓRICAS, METODOLÓGICAS Y PRÁCTICAS
}

\section{UPDATE ON EXPRESSED EMOTION: THEORETICAL, METHODOLOGICAL AND PRACTICAL CONSIDERATIONS}

\author{
Cristina Medina-Pradas \\ Grupo de Investigación "Alteración Mental y Disfunción Social”. \\ Universidad de Sevilla, Sevilla, España
}

Cómo referenciar este artículo/How to reference this article:

Medina-Pradas C. (2016). Actualización en torno a la Emoción Expresada: Consideraciones teóricas, metodológicas y prácticas. Revista de Psicoterapia, 27(103), 251-266.

\section{Resumen}

En el presente trabajo teórico se revisan cuestiones de diferente indole relacionadas con la Emoción Expresada (EE), un campo de trabajo que desde sus inicios a finales de los años sesenta ha llegado a ser muy fructifero para el entendimiento de los factores familiares implicados en los trastornos mentales graves y enfermedades de curso crónico, aunque al que no se le ha dedicado demasiada reflexión crítica. En varios aspectos se hace necesaria una actualización desde una mirada comprehensiva de la literatura que permita avanzar cualitativamente en su estudio, y que repercute en sus aplicaciones clínicas. Primero, se aboga por la consideración de la baja EE y la inclusión de los aspectos positivos, fundamentalmente la calidez, como factores de protección, redirigiendo el tema hacia la psicología positiva. Segundo, se subraya la naturaleza bidireccional y diádica de las relaciones entre pacientes y sus familias en torno a la EE, con las consecuentes implicaciones metodológicas y prácticas a nivel de medida, diseño de estudios y trabajo clínico.

Palabras clave: Familia, Psicopatología, Estrés, Calidez, Estudio Teórico.

\begin{abstract}
In this theoretical article, issues of different nature related to Expressed Emotion (EE) have been reviewed. From its origins in the late 1960s, EE has become a very fruitful field of work for the understanding of the family factors involved in severe mental disorders and chronic illnesses, although without dedicating it enough critical thinking. An update from a comprehensive look at the literature is needed in several aspects in order to progress in their study qualitatively, which will have also important effect on the clinical work. First, it is called for consideration of the low EE and the inclusion of positive aspects, mainly the warmth, as protective factors, redirecting this subject into the framework of the positive psychology. Second, the bidirectional and dyadic nature of relationships between patients and their families around the EE is underlined, with the consequent methodological and practical implications regarding measurement, studies design, and clinical work.
\end{abstract}

Keywords: Family, Psychopathology, Stress, Warmth, Theoretical Study. 


\section{Introducción}

Las teorías sobre el origen social de la enfermedad mental han sido variadas. Durante los años 50 y principios de los 60, predominaban visiones culpabilizadoras de la familia como causante de la enfermedad. En 1975, su validez fue totalmente descartada por Hirsch y Leff cuando al revisarlas concluyen que ninguna identificaba una anormalidad parental característica y específica de las familias de pacientes con un diagnóstico psiquiátrico. Como consecuencia, comenzó la insistencia en desculpabilizar a las familias; no obstante, aunque las teorías "familiogenéticas" de la enfermedad mental sean hoy indefendibles, la importancia de considerar a la familia de los pacientes con la debida atención es innegable.

Los patrones de comunicación familiar han sido siempre objeto de mucha atención en relación a la psicopatología. En parte gracias al desarrollo de instrumentos objetivos de evaluación hacia finales de los 60, se han podido definir y medir algunos aspectos de estos patrones, como por ejemplo la Emoción Expresada (EE) (Brown, Birley y Wing, 1972; Brown y Rutter, 1966). En un principio se intentó averiguar si la EE influía sobre el curso de la esquizofrenia y se descubrió que los pacientes que convivían con un familiar crítico o emocionalmente sobreimplicado recaían con mayor probabilidad. Tras el alta hospitalaria, los pacientes que volvían a un hogar con alta EE tenían un riesgo de recaída del 50\% en los nueve meses siguientes, tasa que bajaba al $15 \%$ en los casos de familias de baja EE (Bebbington y Kuipers, 1994). Entre los pacientes que vivían en hogares con alta EE, el riesgo de recaer se incrementaba hasta un $60 \%$ en aquellos casos en que el contacto cara a cara superaba las 35 horas semanales, disminuyendo hasta el $28 \%$ cuando era menor. Así, al sumar los efectos del no cumplimiento farmacológico con el elevado contacto cara a cara en familias de alta EE, el porcentaje de recaídas se incrementaba hasta el 92\%. El primer descubrimiento lo hicieron Vaughn y Leff (1976a) y desde entonces se han producido réplicas por todo el mundo y en distintas culturas, así como con una gran variedad de diagnósticos psiquiátricos y enfermedades físicas (Bhugra y MacKenzie, 2003; Butzlaff y Hooley, 1998; Hooley, 2007; Wearden, Tarrier, Barrowclough, Zastowny y Amstrong, 2000). De hecho, hoy puede afirmarse que la alta EE familiar influye significativamente en el curso de las enfermedades mentales y físicas de curso crónico, como trastornos afectivos (Ellis et al., 2014), trastornos de ansiedad (Koujalgi, Nayak, Patil y Chate, 2014), trastornos alimentarios (Anastasiadou, Medina-Pradas, Sepulveda y Treasure; 2014), demencia (Li y Murray, 2015), síndrome de fatiga crónica (Band, Barrowclough, y Wearden, 2014), dolor crónico (Ballús-Creus, Rangel, Peñarroya, Pérez y Leff, 2014), entre otras. Por tanto, incluir las intervenciones familiares centradas en la EE en los procesos terapéuticos se ha vuelto primordial.

A pesar de haber ganado mucha popularidad el tema, se considera que está algo encallado. Revisiones sistemáticas recientes han resaltado la necesidad de avanzar conceptual y metodológicamente al observar vacíos teóricos y dificultades que surgen al comparar los estudios (Amaresha y Venkatasubraman, 2012; Duclos, 
Vibert, Mattar y Godart, 2012). Además, se han producido avances que requieren ser considerados. Por tanto, el primer objetivo del presente artículo teórico (Montero y León, 2007) fue llevar a cabo una reflexión comprehensiva y crítica del tema de la EE, y el segundo objetivo fue actualizar cuestiones relacionadas con la medida, proponiendo mejoras en ambos sentidos y dirigidas al trabajo clínico con las familias.

\section{Conceptos relacionados con la Emoción Expresada}

La EE es un concepto que aparece en un contexto de investigación pero que tiene fuertes implicaciones para el trabajo clínico con las familias. Hace referencia a los juicios de contenido afectivo o actitudes emocionales que una persona, en general un familiar o allegado, dirige a otro miembro de la familia con una enfermedad crónica. Es un concepto desarrollado como una medida de la calidad de la relación entre el paciente y el familiar.

Medir la EE es una técnica de investigación, por lo que los componentes del constructo (comentarios críticos, hostilidad y sobreimplicación emocional-SiEson los más usados) son términos técnicos, no nombres de emociones. Se entiende por crítica o comentarios críticos los comentarios desfavorables emitidos sobre la personalidad o conducta del paciente, que a su vez pueden ser clasificados en categorías según a lo que van dirigidos; por hostilidad se entiende la generalización de los comentarios críticos a diferentes áreas, es la crítica o rechazo de la persona en global más que de aspectos concretos de su comportamiento. Y, por $\mathrm{SiE}$ se entiende una respuesta emocional exagerada, de desesperanza, de autosacrificio, sobreidentificación y conducta sobreprotectora extrema expresada por parte del cuidador hacia el paciente.

Sin embargo, existen emociones claramente identificables que son las que dan lugar a las respuestas y conductas que mide la EE. Así, la crítica es una expresión directa del enojo y la rabia, y el número de comentarios críticos registrados durante una entrevista indica el grado de enfado que el cuidador tiene con los comportamientos del paciente. La hostilidad, como forma extrema de crítica, representa un enfado muy intenso, que puede ir acompañado por un rechazo global del paciente, ya no tanto de sus conductas sino de su persona. La SiE está alimentada por una mezcla de ansiedad y culpa. La culpa proviene de sentirse responsable de la enfermedad del paciente y es mucho más común entre los padres que entre otros familiares o cuidadores profesionales. Este sentimiento de culpa lleva a los familiares a hacer todo lo posible por ayudar al paciente para compensar el impacto de la enfermedad en la vida de éste, olvidándose incluso de sus propias necesidades. Por desgracia, esto, lejos de incrementar su autonomía, lleva a que los pacientes se vuelvan cada vez más dependientes de sus familiares y más preocupados por la incapacidad de valerse por sí mismos. Por este motivo, en una relación de $\mathrm{SiE}$ la perspectiva de separarse crea una gran ansiedad en ambas partes.

Por último, existirían determinadas características que pueden estar relaciona- 
das con las emociones anteriores, jugando el papel de posibles determinantes de la EE, aunque el papel predictor de la EE parece ser independiente de casi todos los factores con los que se ha intentado relacionar (Nuechterlein, Snyder y Minz, 1992). Estas características pueden provenir del paciente, de los propios familiares y/o del trastorno en si (Gonzalo y Amor, 2003; Muela y Godoy, 1997; Perez-Pareja, Quiles, Romero, Pamies-Aubalat y Quiles, 2014).

\section{Marco teórico tradicional: estrés y recaída}

Entre los factores que explicarían el desencadenamiento de muchas de las enfermedades mentales dentro de una teoría multifactorial de su etiología se halla la mayor vulnerabilidad al estrés del individuo genéticamente predispuesto a la enfermedad. En 1977, Zubin y Spring desarrollaron el modelo de vulnerabilidadestrés, que ha servido de guía y marco conceptual. En esta línea se han intentado estudiar los marcadores conductuales de la vulnerabilidad personal en el contexto de los estresantes ambientales tanto intra como extrafamiliares. Según estos autores, para que aparezca la enfermedad es necesaria la acción de un factor estresante sobre un organismo con cierta vulnerabilidad y, a mayor vulnerabilidad, menos estrés será necesario para que se produzca la enfermedad, y viceversa. En consecuencia, el control del estrés aparece como una forma de reducir la tasa de recaídas, o incluso evitar la aparición de enfermedad.

Es importante especificar al máximo los marcadores de vulnerabilidad potenciales y las condiciones de los estresores que pueden asociarse a la aparición y curso de la enfermedad. Por un lado, los estudios de alto riesgo se han centrado en los marcadores de vulnerabilidad individual; por otro, están los estudios concentrados en determinar factores estresantes. Concretamente, el estrés asociado a los acontecimientos vitales (estrés agudo delimitado en el tiempo) y el nivel de estrés familiar (que para aquellos pacientes que viven con sus familiares puede llegar a ser un estrés crónico, continuo). En este sentido, Leff, Kuipers, Berkowitz, Vaughn y Sturgeon (1983) afirmaron que los pacientes son vulnerables a estos dos tipos de estrés: al agudo, que actuaría como precipitante y no está bajo control, y al crónico, cuya modificalidad lo convierte en el principal foco de interés. Los estudios han ido desarrollándose según esta línea con el objetivo de obtener y analizar información adicional sobre los mecanismos a través de los cuales la EE juega su papel de máximo agente del ambiente familiar como fuente de estrés crónico y como mejor predictor del pronóstico (Hooley y Gotlib, 2000).

Hay varias líneas de evidencia que indican que alta EE familiar representa un significante estresor para los pacientes con diversos trastornos hasta el punto de poder precipitar recaídas. Primero, una serie de estudios fisiológicos (Tarrier y Turpin, 1992) demuestran que los niveles de arousal de los pacientes difieren en respuesta a alta y baja EE. La presencia de un pariente de alta EE se asocia con mantenimiento o incremento del arousal, y la de un pariente de baja EE con el descenso del arousal. Además, la combinación de altos niveles de EE en la familia 
y la activación del sistema nervioso simpático que ocurre en los pacientes confiere un riesgo especial de cara a un peor pronóstico sintomático (Subotnik et al., 2012). Segundo, estudios de interacción entre familiares y pacientes en el laboratorio han encontrado diferencias en familiares de alta y baja EE y se ha comprobado que estos resultados son generalizables a la vida real (Cruise, Sheeber, y Tompson, 2011; Hahlweg, Goldstein y Nuechterlein, 1989). Se observaron más comportamientos intrusivos en los familiares de alta $\mathrm{EE}$, quienes tuvieron más dificultades para cambiar situaciones negativas derivadas de comportamientos problemáticos de los pacientes, siendo sus respuestas más tendentes a contribuir a un escalamiento de intercambios negativos.

Investigaciones más recientes centradas en EE siguen apoyando este modelo de vulnerabilidad-estrés y la hipótesis de la EE como estresor desencadenante de recaídas (Amaresha y Venkatasubramanian, 2012; Duclos et al., 2012). Los pacientes preguntados directamente informan de incrementos significativos en ansiedad, ira y estrés después de ser expuestos a alta EE (Finnegan et al., 2014). Más concretamente, éstos se sienten más estresados cuando se encuentran interactuando con familiares críticos que ante aquellos sobreimplicados emocionalmente, aun sintiendo estrés ante ambos a diferencia de ante familiares cálidos (Medina-Pradas, Navarro, López, Grau y Obiols, 2011a). Esos niveles de estrés a su vez están influenciados por su sensibilidad a las críticas (Cutting, Aakre y Docherty, 2006) y sus niveles de ansiedad, los cuales interactuarían en última instancia con la EE en la exacerbación de los síntomas (Docherty et al., 2011). Además, la EE puede influir en la aparición de un trastorno como desencadenante relacionado con el estrés (Treasure y Schmidt, 2013).

\section{Hacia una visión más positiva de la EE}

Llegados a este punto, surgen las siguientes cuestiones: ¿qué ha pasado con los componentes positivos de la EE?, ¿qué ocurre con los familiares de baja EE? La expresión "EE alta" es un tanto equívoca (Kuipers et al., 2002), ya que a menudo da lugar a que se interprete preferible que los familiares supriman cualquier emoción en presencia del paciente. Para evitar malentendidos, hubiese sido preferible utilizar la etiqueta "EE negativa alta", pero el término acuñado por sus creadores Brown y Rutter está ya demasiado arraigado. Como se ha demostrado ampliamente, las emociones negativas (crítica, hostilidad y $\mathrm{SiE}$ ) empeoran la evolución de las personas con algún trastorno mental y deberían evitarse. Sin embargo, la presencia de calidez y comentarios positivos (los otros componentes, por lo general olvidados, que componen los aspectos positivos de la EE) se está viendo recientemente que se relacionan con menor severidad de los síntomas (Medina-Pradas et al., 2011a; Medina-Pradas, Navarro, Pousa, Montero y Obiols, 2013) y mejor funcionamiento (Schlosser et al., 2010), mejoran el pronóstico de la enfermedad funcionando como factores protectores de recaídas (Lee, Barrowclough y Lobban, 2014; López et al., 2004) e, incluso, protegen de la aparición de 
enfermedad mental (González-Pinto et al., 2011). Todos estos resultados nos dirigen hacia el irrefutable camino de incluir los aspectos positivos de la EE y considerar nuevos marcos teóricos.

Por otro lado, estudios cualitativos han analizado a los familiares de EE baja, para confirmar que éstos no son simplemente neutros (Treanor, Lobban y Barrowclough, 2011; Treanor, Lobban y Barrowclough, 2013). No se caracterizan únicamente por no ser críticos, hostiles o sobreimplicados, sino que se identifican con una serie de estrategias de afrontamiento positivas. Aunque también describen experiencias estresantes y sentimientos de frustración e ira, muestran empatía y compromiso para apoyar al enfermo, demuestran comprensión hacia la enfermedad y los comportamientos relacionados y han ajustado sus expectativas de futuro. La diferencia fundamental con los familiares de EE alta es su actitud hacia los síntomas y la conducta del paciente, su reacción ante los mismos hechos. Por ejemplo, mientras los familiares de EE alta descalifican las experiencias del paciente, los familiares de EE baja reconocen la realidad de sus experiencias, pero dejando claro que no las comparten.

Por ello, uno de los objetivos del trabajo clínico con familias con un miembro que padece una enfermedad crónica debe ser incrementar tanto las actitudes positivas por parte de los familiares (calidez y comentarios positivos) como las actitudes típicas de los familiares de baja EE de cara a construir fortalezas dentro de la familia y promover mejores formas de responder al ser querido. Todo esto ayudará a promover el bienestar psicológico de los familiares y de los propios pacientes y, en consecuencia, los protegerá frente a posibles recaídas mejorando sus pronósticos.

En definitiva, y como además vienen apuntando las últimas revisiones de la literatura sobre EE (Amaresha y Venkatasubramanian, 2012), se vuelve fundamental considerar estos aspectos positivos a la hora de evaluar y trabajar con la EE, tanto en clínica como en investigación. Los modelos prevalentes de la asociación EErecaída conceptualizan la EE como una forma de estrés para los pacientes, básicamente en base al modelo de vulnerabilidad-estrés, siendo hora de integrar los aspectos positivos de la EE incluyendo modelos teóricos basados en la psicología positiva (Fernandez-Ríos y Novo, 2012), como el de Fredickson (Fredickson, 2001), y enfocar su estudio no sólo como factor de riesgo sino también como factor protector.

\section{Considerando la naturaleza bidireccional y diádica de la EE}

Miklowitz (2004), en su análisis de la EE dentro de la teoría sistémica y en base a conceptos provenientes de la investigación en psicopatología del desarrollo, apuntó que las influencias paciente-familiares son bidireccionales, es decir, no sólo influye la EE y el estrés familiar sobre el paciente y el curso de su trastorno, sino también influyen las características del paciente y su trastorno sobre la familia. Además, EE y trastorno cambian al mismo tiempo, y las actitudes relacionadas con 
la EE se asocian con ciclos de interacción mutuamente influyentes entre los familiares y los pacientes (Burbach, 2013), no sólo refleja alteraciones en la organización, clima emocional y patrones transaccionales del sistema familiar. Por ello, las alteraciones subyacentes en los sistemas familiares pueden emerger en respuesta a los síntomas de la enfermedad en uno de sus miembros y tener efectos recurrentes en el desarrollo y curso de la enfermedad una vez manifestada. Asimismo, la naturaleza y estabilidad de estos efectos recursivos dependen de procesos dinámicos en el paciente, en el familiar y en su relación.

Por otro lado, el modelo de Lazarus y Folkman (Lazarus, 1993; Lazarus y Folkman, 1984) considera que entre las circunstancias objetivas y las respuestas psicológicas están las valoraciones personales que los sujetos hacen de esas circunstancias. Al aplicar este modelo a los procesos que rodean la $\mathrm{EE}, \mathrm{y}$ considerando los apuntes de Miklowitz, nos preguntamos cómo los pacientes perciben la EE de sus familiares, de lo que sorprende lo poco que se sabe sobre ello a pesar de ser ellos las dianas de esas actitudes (Renshaw, 2008).

Las percepciones de los pacientes podrían funcionar como una manera más rápida y fácil de capturar la esencia de la $\mathrm{EE}$, pudiendo ayudar en el avance del entendimiento que tenemos de los mecanismos transaccionales y las dinámicas familiares que se generan alrededor del fenómeno de la EE. Con esta información complementaria, obtendríamos una evaluación balanceada de la díada, es decir, no sólo nos quedaríamos con la perspectiva de los familiares, sino que avanzaríamos hacía una visión diádica de la EE. Con esto, además, se enfatiza la importancia de la perspectiva desde la que es medida la EE: si es una visión objetiva, evaluada por un observador externo entrenado en EE; o es la de un informador interno, una perspectiva autoevaluada y subjetiva (los propios familiares); o si es la de un informador interno, también subjetiva, pero percibida (la de los pacientes). La consideración de cada una de estas perspectivas tendría sus implicaciones. Muy pocos estudios han considerado la perspectiva de familiares y pacientes a la vez, aunque los que lo han hecho han demostrado que ambas no coinciden en condiciones generales (Bachmann, Bottmer, Jacob y Schröder, 2006; Cutting et al., 2006; Di Paola, Faravelli y Ricca, 2010; Kazarian, Maya, Cole y Baker, 1990; MedinaPradas et al., 2013; Moulds et al., 2000; Perkins et al., 2005; Tompson et al., 1995; Scazufca, Kuipers y Menezes, 2001; Weisman, Rosales, Kymalainen y Armesto, 2006), datos también a favor de la necesidad de considerar ambas.

Se ha visto además que las percepciones de la crítica parecen ser útiles para predecir el curso futuro de los síntomas (Miklowitz, Wisniewski, Miyahara, Otto y Sachs, 2005), aunque más datos hacen falta para confirmar este hallazgo. Lo que ocurre es que a los pacientes tradicionalmente no se les ha preguntados ni por cómo perciben la EE ni por el estrés que les genera fundamentalmente porque no existían medidas específicas y completas para ello. Para la EE percibida, aparte de la "Escala de crítica percibida" (Perceived Criticism Scale, PCS; Hooley y Teasdale, 1989), se conoce la "Escala del nivel de emoción expresada" en su versión para pacientes 
(Level of expressed emotion, LEE; Cole y Kazarian, 1988), aunque ésta no está basada en el constructo de EE directamente -no mide específicamente SiE o crítica, sino aspectos relacionados (Hooley y Parker, 2006). Por tanto, no es hasta 2011 que surge la "Escala diádica breve de emoción expresada" (BDSEE), con una versión para pacientes (Medina-Pradas, Navarro, López, Grau y Obiols, 2011b) y otra para familiares (Medina-Pradas, Domínguez-Martínez, Kwapil, BarrantesVidal y Lopez, sometido a publicación), la cual no sólo incluye la visión diádica, sino también los aspectos positivos de la EE, concretamente la calidez, conformándose como una herramienta ideal para el trabajo con la EE desde la perspectiva más moderna por la que se está abogando. Junto a ella, la "Escala de estrés percibido debido a la emoción expresada" (PSEE; Medina-Pradas et al., 2011a) que mide el estrés percibido de los pacientes ante la EE de sus familiares (en concreto ante la crítica, la $\mathrm{SiE}$ y la calidez), completarían la información necesaria para tener el cuadro completo. Hablaremos de ellas en el siguiente apartado.

Para finalizar, resulta práctico ampliar el constructo de la EE hacia ciclos de comunicación útiles en el trabajo psicoterapéutico con los pacientes y sus familias, derivados de la reflexión sobre los resultados obtenidos en las investigaciones y a la luz de este nuevo planteamiento bidireccional y diádico. Los objetivos terapéuticos serían reducir los niveles de crítica y $\mathrm{SiE}$, aumentar los niveles de calidez, y considerar las percepciones por parte de los pacientes de la EE y las valoraciones subjetivas que los familiares hacen de la enfermedad. El fin sería ver si es necesario desactivar circuitos recursivos, bucles mantenedores de conflicto, o reconstruir/ reestructurar dichas valoraciones por alternativas más útiles para la familia, observando además si dentro del subsistema parental las construcciones personales de la enfermedad entran en conflicto agravando la situación (por ejemplo, entre familiares críticos y sobreimplicados).

Para presentar estos ciclos de comunicación, retomaremos la noción de nudo del problema de Procter (Procter, 1981, 1985) y se modelizarán los circuitos recursivos de la crítica y la $\mathrm{SiE}$ mediante diagramas de la posición de cada persona implicada en la situación. Procter usa la teoría de los constructos personales como base para una teoría del sistema de constructos familiares (Feixas, 1998), lo cual proporciona un marco excelente para explicar las interacciones familiares y, en nuestro caso, para poder visualizar la EE desde esta nueva visión por la que estamos abogando. La noción de posición de Procter supone dos niveles: el nivel de construcción (cómo construye uno la actitud del otro -el significado que da a sus percepciones) y el nivel de acción (qué conducta sucede a dicha construcción).

Así, el primer modelo correspondiente a la crítica (ver Figura 1), representa cómo la actitud del cuidador (lo que tradicionalmente se ha venido observando, es decir, la EE del cuidador, en este caso, los comportamientos críticos) genera en el paciente construcciones del tipo "me odia", "solo quiere controlar mi vida", etc. (serían las percepciones del pacientes de dicha EE), lo que generaría a efectos conductuales una actitud de desafío, de atrincheramiento en los síntomas, llegando 
Figura 1

DIAGRAMA DE POSICIÓN (I): Nudo del Problema: LA CRÍTICA

\section{El punto de vista del paciente El punto de vista del cuidador}

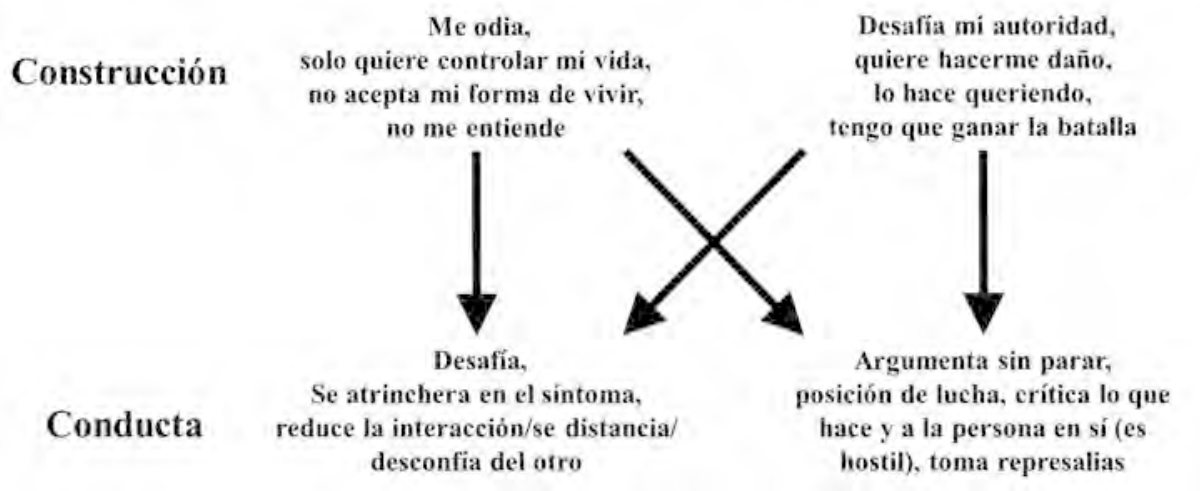

a distanciarse del cuidador y a desconfiar de él (cómo se reflejarían sus percepciones de la EE en su conducta - lo que incluiría los síntomas, su funcionamiento, la enfermedad en definitiva). Y esto, a su vez, generaría en el cuidador la construcción de "mi hijo desafía mi autoridad", "lo hace todo para molestarme porque podría controlar más", etc. (estaríamos hablando de las valoraciones subjetivas del cuidador), conduciéndole a argumentar incansablemente adoptando una posición de lucha, crítica y hasta hostilidad con frecuentes represalias (la EE), lo que volvería a retroalimentar la construcción en el paciente reactivando el circuito recursivo en el que se debería intervenir. Y el estrés estaría mediando entre las construcciones y las conductas de ambos.

De igual manera puede representarse la $\mathrm{SiE}$ (ver Figura 2). El paciente construiría dicha $\mathrm{SiE}$, por una lado, considerando que el trastorno le hace especial, le da poder; y, por otro lado, pensando que el mundo es un lugar peligroso y él/la una persona débil que debe ser protegida. Esta construcción conduciría a perpetuar los síntomas de fragilidad, a ver minada su autoestima, a ser cada vez más dependiente e infantilizado, y a aislarse del grupo de iguales, lo que generaría en el cuidador la sensación de tener que hacer todo lo que pueda para que su hijo/a no empeore, conduciendo a una actitud de constante autosacrificio, comprobación ansiosa de que está bien, intrusismo y a perder otros roles sociales incompatibles con esta dedicación exclusiva. La visión de estas actitudes volvería a retroalimentar la construcción del paciente retroalimentando de nuevo el circuito.

Y lo mismo podríamos hacer con la calidez. Por tanto, puede decirse que conceptualizar los procesos familiares que rodean la EE como construcciones y acciones unidas entre sí en secuencias interaccionales permite a los terapeutas tener más información para la intervención y para generar (re)construcciones alternativas que ayuden a las familias. 


\section{Figura 2}

DIAGRAMA DE POSICIÓN (il): Nudo del Problema: LA SiE

El punto de vista del paciente El punto de vista del cuidador

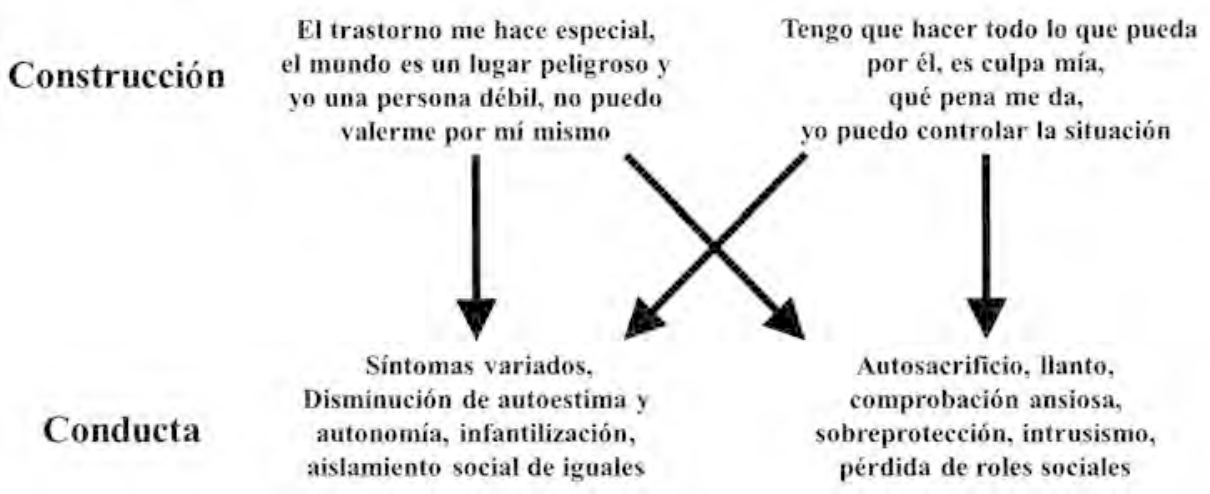

\section{Evaluación de la EE}

A continuación, se comentan las ventajas e inconvenientes de los instrumentos de evaluación de la EE más utilizados, con el objetivo de sacar conclusiones sobre los más útiles a la luz de las nuevas tendencias. Para una revisión exhaustiva, consultar Becerra (2011).

La entrevista gold-standard creada específicamente para evaluar la EE en familiares es la "Entrevista familiar Camberwell" (Camberwell family interview, CFI; Vaughn y Leff, 1976b). Es una entrevista semiestructurada, de hora y media aproximadamente de duración, que evalúa comentarios críticos, hostilidad, SiE, calidez y comentarios positivos. El período de tiempo que evalúa la CFI son los tres meses previos. Una familia será considerada como de alta $\mathrm{EE}$ a partir de que alguno de sus miembros supere el punto de corte en crítica y/o en SiE y/o presente hostilidad. Tiene ventajas claras, fundamentalmente la riqueza de la información que permite recoger, la cual puede usarse desde un punto de vista tanto cualitativo como cuantitativo. No obstante, no es tan ventajosa cuando hablamos de los recursos temporales, personales y económicos que requiere para su aplicación y corrección. La CFI se muestra excesivamente larga y complicada porque debe ser codificada a posteriori por dos evaluadores independientes. Para ello, es necesaria una formación específica de los evaluadores con la autora Christine Vaughn en Londres para obtener la fiabilidad en su uso -en España, también es posible obtener esta certificación con Cristina Medina-Pradas y Adrián Montesano, formados y autorizados por la Dra. Vaughn. Por último, el punto de corte de la CFI resulta en ocasiones artificioso (por ejemplo, en crítica se han utilizado como criterios 4, 6, 7 ó 9, según el estudio). Por tanto, a pesar de ser el instrumento que proporciona la información más rica y de considerarse el de elección en la medida de lo posible, 
la mayoría de las veces y sobre todo en clínica se han de considerar otros métodos más factibles (aunque de cara a interpretar los resultados de la investigaciones y a trabajar en terapia con las familias se siga recomendando la formación reglada).

Un intento de superar estos inconvenientes sería utilizar una medida más corta y fácil de aplicar y evaluar. Una entrevista alternativa no estructurada es la "Muestra del discurso de cinco minutos" (Five Minute Speech Sample, FMSS; Magana et al., 1986), aunque ésta también requiere entrenamiento y acuerdo entre observadores. Cada familiar debe hablar durante cinco minutos sobre el tipo de persona que considera que es el paciente y el tipo de relación que tienen. Se codifican crítica, hostilidad y $\mathrm{SiE}$ (no los aspectos positivos de la EE, lo cual apunta el primer inconveniente), considerándose que una familia presenta $\mathrm{EE}$ alta si el comentario inicial es negativo, si la relación es valorada como negativa, si se realizan uno o más comentarios críticos, si existe evidencia de autosacrificio o comportamiento sobreprotector, si se realizan manifestaciones emocionales a lo largo de la entrevista o si se dan excesivos detalles sobre el pasado. Otro inconveniente lo apuntaron Parker y Johnston (1988) al encontrar que familiares que puntúan en baja EE con la FMSS obtenían puntuaciones significativamente más altas en la escala de falseamiento de una medida de personalidad en comparación con los familiares definidos como de alta EE. Concluyeron que deberían requerirse estrategias correctivas para controlar dicho efecto de deseabilidad social que estaría modificando las subsecuentes asignaciones a un grupo u otro de EE en estudios que utilizaran esta medida. Además, el tiempo de aplicación de la FMSS es valorado como extremadamente escaso de cara al descenso de la posible reactividad a la situación de evaluación. Haldford (1992) concluyó que la FMSS correlaciona altamente con la CFI en términos de clasificación de familias como de alta o baja EE pero tiende a puntuar en menor medida la ocurrencia de alta EE. Por estas razones, algunos autores asignan los sujetos de baja EE con puntuaciones límite al grupo de alta EE, demostrando que mejora la sensibilidad y la validez de la prueba. En definitiva, la FMSS no parece ser una buena alternativa para evaluar la EE.

Hay también otras formas de evaluar los índices negativos de la EE mediante escalas autoaplicables, como por ejemplo, la ya comentada $\boldsymbol{L E} \boldsymbol{E}$ (Cole y Kazarian, 1988), que por su brevedad y fácil aplicación y corrección palian algunas de las limitaciones de las entrevistas comentadas. Sin embargo, como se expuso anteriormente, sólo hay un instrumento, la BDSEE (Medina-Pradas et al., 2011b; MedinaPradas et al., en revisión), que, además de fácil de aplicar y corregir, incluye los aspectos positivos y la visión diádica de la EE, conformándose como la medida más completa de la EE junto con la PSEE (Medina-Pradas et al., 2011a). Previamente a la existencia de la BDSEE, las revisiones sobre la medición de la EE venían a concluir que las pruebas tipo entrevista presentaban limitaciones que reducían su aplicabilidad clínica y que las pruebas breves requerían de mayor investigación y examen conceptual (Becerra, 2011; Hooley y Parker, 2006; Van Humbeeck, Van Audenhove, De Hert, Pieters y Storms, 2002), sin decantarse por ninguna en 
concreto. Así, actualmente puede recomendarse el uso de la BDSEE y la PSEE como medidas de elección, invitando asimismo a continuar la investigación con ellas en algunos aspectos pendientes (por ejemplo, replicar resultados con otros grupos diagnósticos o evaluar su validez predictiva). Ambas se encarnarían como la mejor manera para medir la EE hasta el momento, alternativa psicométricamente fuerte, conceptualmente completa y muy práctica tanto para contextos clínicos como de investigación.

\section{Conclusiones}

Tras la revisión comprehensiva y crítica de la literatura sobre EE y en relación a los dos objetivos marcados, se extraen dos conclusiones fundamentales que a partir de ahora deberían tenerse en cuenta a la hora de investigar y trabajar con la EE. Primero y en relación a aspectos más teóricos, incluir los aspectos positivos de la EE como factores de protección y a promover en las familias, junto con los negativos, enmarcando el estudio de la EE en un contexto de psicología más positiva. Segundo y a un nivel más metodológico, no olvidar la perspectiva de los pacientes sobre la EE y el estrés que les produce, considerando la visión diádica y completa del fenómeno dinámico de comunicación bidireccional producido en torno a la EE, lo que implica a su vez un cambio en los instrumentos de medida y en los diseños de estudio.

A nivel clínico, demostrado que la familia es un agente activo de cambio y posible factor protector (y no sólo promotor de recaídas), habría que trabajar con ella desde una concepción de co-construir nuevas formas de relacionarse con el paciente y su trastorno. Las líneas que pueden seguirse son desde reducir el estrés emocional en el contexto familiar como factor de mantenimiento y agravación de la enfermedad hasta la promoción del afecto positivo en las interacciones familiares de cara a mejorar el pronóstico. Es decir, debemos intervenir también la EE desde un contexto más actual relacionado con la psicología positiva y la promoción de la salud en lugar de meramente con la vulnerabilidad al estrés y la prevención de empeoramiento. Las intervenciones familiares y/o multifamiliares son ambientes privilegiados de trabajo (Gutiérrez, Sepulveda, Anastasiadou y Medina-Pradas, 2014; Pharoah, Mari, Rathbone y Wong, 2010), que asimismo deberían considerar tanto la visión de los familiares como la de los pacientes en su relación.

\section{Agradecimientos}

Este trabajo se ha generado como consecuencia de varios años de trabajo de la autora con familias de diferentes tipos de pacientes, inicialmente desde la Universidad Autónoma de Barcelona bajo el apoyo del Programa de Formación de Personal Investigador del Ministerio de Economía y Competitividad (BSO200305561), y posteriormente desde la Universidad Autónoma de Madrid (Programa Postdoctoral Alianza 4 Universidades). A todas estas familias, gracias por su generosidad. La autora agradece también el importante apoyo de determinados 
colegas en diferentes momentos de este proceso (Dr. Jordi Obiols, Dra. Isabel Montero, Dra. Elizabeth Kuipers, Dra. Christine Barrowclough, Dr. Steven López y Dr. Adrián Montesano), en especial a la Dra. Christine Vaughn.

\section{Referencias bibliográficas}

Amaresha, A.C. y Venkatasubramanian, G. (2012). Expressed Emotion in Schizophrenia: An Overview. Indian Journal of Psychological Medicine, 34, 12-20.

Anastasiadou, D., Medina-Pradas, C., Sepulveda, A.R. y Treasure, J. (2014). A systematic review of family caregiving in eating disorders. Eating Behaviours, 15(3), 464-477.

Bachmann, S., Bottmer, C., Jacob, S. y Schröder, J. (2006). Perceived criticism in schizophrenia: A comparison of instruments for the assessment of the patient's perspective and its relation to relatives' EE. Psychiatry Research, 142, 167-175.

Ballús-Creus, C., Rangel, M.V., Peñarroya, A., Pérez, J. y Leff, J. (2014). Expressed emotion among relatives of chronic pain patients, the interaction between relatives' behaviours and patients' pain experience. International Journal of Social Psychiatry, 60(2), 197-205.

Bebbington, P. y Kuipers, L. (1994). The predictive utility of expressed emotion in schizophrenia: an aggregate analysis. Psychological Medicine, 21, 707-718.

Becerra, J.A. (2011). Descripción y limitaciones de los instrumentos de evaluación de la emoción expresada. Papeles del Psicólogo, 32, 152-158.

Bhugra, D. y McKenzie, K. (2003). Expressed Emotion across cultures. Advances in Psychiatric Treatment, 9, $342-$ 348.

Brown, G.W.y Rutter, M. (1966). The measurement of family activities and relationships: a methodological study. Human Relations, 19, 241-263.

Brown, G.W., Birley, J.L.T. y Wing, J.K. (1972). Influence of family life on the course of schizophrenic disorders: a replication. British Journal of Psychiatry, 121, 241-258.

Burbach, F.R. (2013). Towards a systemic understanding of family emotional atmosphere and outcome after psychosis. En A. Gumley, A. Gillham, K. Taylor y M. Schwannauer(eds.), Psychosis and Emotion (pp. 116235). USA y Canada: Routledge.

Butzlaff, R.L. y Hooley, J.M. (1998). Expressed emotion and psychiatric relapse. Archives of General Psychiatry, 55, 547- 552 .

Cole, J.D. y Kazarian, S.S. (1988). The level of expressed emotion scale: a new measure of expressed emotion. Journal of Clinical Psychology, 44, 392-397.

Cruise, R.C., Sheeber, L.B. y Tompson, M.C. (2011). Behavioural correlates of maternal expressed emotion in interaction tasks. Journal of Family Psychology, 25(5): 781-784.

Cutting, L.P., Aakre, J.M. y Docherty, N.M. (2006). Schizophrenic Patients' Perceptions of Stress, Expressed Emotion, and Sensitivity to Criticism. Schizophrenia Bulletin, 32(4), 743-750.

Dean, N. y Lin, N. (1977). The stress-buffering role of social support. Journal of Nervous and Mental Disease, 165, 403-416.

Di Paola, F., Faravelli, C. y Ricca, V. (2010). Perceived expressed emotion in anorexia nervosa, bulimia nervosa, and binge-eating disorder. Comprehensive Psychiatry, 51, 401-405.

Docherty, N.M., St-Hilaire, A., Aakre, J.M., Seghers, J.P., McCleery, M. y Divilbiss, M. (2011). Anxiety Interacts With Expressed Emotion Criticism in the Prediction of Psychotic Symptom Exacerbation. Schizophrenia Bulletin, 37(3), 611-618.

Duclos, J., Vibert, S., Mattar, L. y Godart, N. (2012). Expressed Emotion in Families of Patients with Eating Disorders: A Review of the Literature. Current Psychiatry Reviews, 8.

Ellis, A.J., Portnoff, L.C., Axelson, D.A., Kowatch, R.A., Walshaw, P. y Miklowitz, D.J. (2014). Parental expressed emotion and suicidal ideation in adolescents with bipolar disorder. Psychiatry Research, 216(2): 213-16.

Feixas, G. (1998). Los constructos personales en la práctica sistémica. En: R.A. Neimeyer, M.J. Mahoney (eds.), Constructivismo en psicoterapia (pp. 273-309). Barcelona: Paidós.

Fernandez-Rios, L. y Novo, M. (2012). Positive Pychology: Zeigeist (or spirit of the times) or ignorance (or disinformation) of history? International Journal of Clinical and Health Psychology, 12(2), 333-344. 
Finnegan, D., Onwumere, J., Green, C., Freeman, D., Garety, P. y Kuipers, E. (2014). Negative communication in psychosis: understanding pathways to poorer patient outcomes. Journal of Nervous and Mental Disorders, 202, 829-832.

Fredickson, B.L. (2001). The role of positive emotions in positive psychology. The Broaden-and-Build Theory of positive emotions. American Psychologist, 56, 218-226.

Gonzalez-Pinto, A., Ruiz de Azua, S., Ibanez, B., Otero-Cuesta, S., Castro-Fornieles, J., Graell-Berna, M., ... Arango, C. (2011) Can positive family factors be protective against the development of psychosis? Psychiatry Research, 186, 28-33.

Gonzalo, N. y Amor, B. (2003). Intervención familiar en la esquizofrenia. En E. Doménech-Llaberia, J.E. Obiols, M.C. Janè y S. Subirà (eds.), Actualizaciones en psicopatología clínica 2003 (pp. 295-332). Barcelona: Masters Psicopatología UAB.

Gutierrez, E. Sepulveda, A.R., Anastasiadou, D. y Medina-Pradas, C. (2014). Programa de psicoeducación familiar para los trastornos del comportamiento alimentario. Behavioral Psychology/Psicologia Conductual, 22(1), 133-149.

Hahlweg, W.K., Goldstein, M.J. y Nuechterlein, K.H. (1989). Expressed emotion and patient relative interaction in families of recent onset schizophrenics. Journal of Consulting and Clinical Psychology, 57, 11-18.

Halford, K.W. (1992). Assessment of family interaction with a schizophrenic member. In D.J. Kavanagh (ed.), Schizophrenia: An overview and practical handbook (pp. 254-274). London: Chapman \& Hall.

Hirsch, S.R. y Leff, J. (1975). Abnormalities in patients of schizophrenics. Maudsley Monograph $n^{\circ} 22$. Londres: Oxford University Press.

Hooley, J.M. (2007). Expressed Emotion and Relapse of Psychopathology. Annual Review of Clinical Psychology, 3, 329-352.

Hooley, J.M. y Gotlib, I.H. (2000). A diathesis-stress conceptualization of expressed emotion and clinical outcome. Applied and Preventive Psychology, 9, 135-151.

Hooley, J.M. y Parker, H.A. (2006). Measuring expressed emotion: an evaluation of the shortcuts. Journal of Family Psychology, 20, 386-396.

Kazarian, S.S., Malla, A.K., Cole, J.D. y Baker, B. (1990).Comparisons of two expressed emotion scales with the Camberwell Family Interview. Journal of Clinical Psychology, 46, 306-309.

Koujalgi, S.R., Nayak, R.B., Patil, N.M. y Chate, S.S. (2014). Expressed emotions in patients with obsessive compulsive disorder: a case control study. Indian Journal of Psychological Medicine, 36(2), 138-141.

Kuipers, E., Leff, J. y Lam, D. (2002). Family Work for Schizophrenia. London: Gaskell.

Lazarus, R.S. (1993). From psychological stress to the emotions: a history of changing outlooks. Annual Review of Psychology, 44, 1-21.

Lazarus, R. y Folkman, S. (1984). Stress, appraisal and coping. Nueva York: Springer.

Lee, G., Barrowclough, C. y Lobban, F. (2014). Positive affect in the family environment protects against relapse in first-episode psychosis. Social Psychiatry and Psychiatric Epidemiology, 49, 367-376.

Leff, J., Kuipers, L., Berkovitz, R., Vaughn, C. y Sturgeon, D. (1983). Life events, relatives expressed emotion and maintenance neuroleptics in schizophrenic relapse. Psychological Medicine, 13, 799-806.

Li, C.Y.y Murray, M. (2015). A review of conceptualisation of expressed emotion in caregivers of older adults with dementia. Journal of Clinical Nursing, 24(3-4): 332-343.

López, S.R., Hipke, K.N., Polo, A.J., Jenkins, J.H., Karno, M., Vaughn, C. y Snyder, K.S. (2004). Ethnicity, expressed emotion, attributions, and course of schizophrenia: Family warmth matters. Journal of Abnormal Psychology, 113, 428-439.

Magana, A.B., Goldstein, M.J., Karno, M., Miklovitz, D.J., Jenkins, J. y Fallon, I.R.H. (1986). A brief method for assessing expressed emotion in relatives of psychiatric patients. Psychiatric Research, 17, 203-212.

Medina-Pradas, C., Domínguez-Martínez, T., Kwapil, T.E., Barrantes-Vidal, N., López, S.R. The Brief Dyadic Scale of Expressed Emotion (BDSEE) - Relatives Version. Manuscrito enviado para publicación. 2016.

Medina-Pradas, C., Navarro, J.B., López, S.R., Grau, A. y Obiols, J.E. (2011a). Dyadic view of expressed emotion, stress, and eating disorder psychopathology. Appetite, 57(3), 743-748.

Medina-Pradas, C., Navarro, J.B., López, S.R., Grau, A. y Obiols, J.E. (2011b). Further development of a scale of perceived expressed emotion and its evaluation in a sample of patients with eating disorders. Psychiatry research, 190(2-3), 291-296.

Medina-Pradas, C., Navarro, J.B., Pousa, E., Montero, M.I. y Obiols, J.E. (2013). Expressed and perceived criticism, family warmth, and symptoms in schizophrenia. Spanish Journal of Psychology, 16(45), 1-8.

Miklovitz, D.J. (2004). The role of family systems in severe and recurrent psychiatric disorders: a developmental psychopathology view. Development and Psychopathology, 16, 667-688. 
Miklowitz, D.J., Wisniewski, S.R., Miyahara, S., Otto, M.W. y Sachs, G.S. (2005). Perceived criticism from family members as a predictor of the one-year course of bipolar disorder. Psychiatry Research, 136, 101-111.

Montero, I. y León, O.G. (2007). A guide for naming research studies in Psychology. International Journal of Clinical and Health Psychology, 7, 847-862.

Moulds, M.L., Touyz, W., Schotte, D., Beumont, P.J.V., Griffiths, R., Russell, J. y Charles, M. (2000). Perceived expressed emotion in the siblings and parents of hospitalized patients with anorexia nervosa. International Journal of Eating Disorders, 27, 288-296.

Muela, J.A. y Godoy, J.F. (1997). El estrés crónico en la esquizofrenia: la emoción expresada. Revista electrónica de Motivación y Emoción, 4, 7.

Nuechterlein, K.H., Snyder, K.S. y Minz, J. (1992). Paths to relapse: possible transactional processes connecting patient illness onset, expressed emotion, and psychotic relapse. The British Journal of Psychiatry, Supp. 18, 88-96.

Parker, G. y Johnston, P. (1988). Examining for links between expressed emotion and EPI personality variables: Can low EE be faked? Acta Psychiatrica Scandinavica, 78, 599-602.

Perez-Pareja, B., Quiles, Y., Romero, C., Pamies-Aubalat, L. y Quiles, M.J. (2014). Malestar psicológico y emoción expresada en cuidadores de pacientes con un trastorno de la conducta alimentaria. Anales de Psicología, 30, $1,37-45$.

Perkins, S., Schmidt, U., Eisler, I., Treasure, J., Yi, I., Winn, S., ... Berelowitz, M. (2005). Why do adolescents with bulimia nervosa choose not to involve their parents in treatment? European Child and Adolescent Psychiatry, 14, 376-385.

Pharoah, F., Mari, J.J., Rathbone, J., Wong, W. (2010). Family intervention for schizophrenia. Chochrane Database of Systematic Reviews, 12: Art. N ${ }^{\circ}$ CD000088.

Procter, H. (1981). Family construct psychology: An approach to understanding and treating families. En: S. Walrond-Skinner (ed.), Developments in family therapy: Theories and applications since 1948. Londres: Routledge y Kegan Paul.

Procter, H. (1985). A personal construct approach to family therapy and systems intervention. En E. Button (ed.), Person construct theory and mental health. Londres: Croom Helm.

Renshaw, K.D. (2008). The predictive, convergent, and discriminant validity of perceived criticism: A review. Clinical Psychology Review, 28, 521-534.

Scazufca, M., Kuipers, E. y Menezes, P.R. (2001). Perception of negative emotions in close relatives by patients with schizophrenia. British Journal of Clinical Psychology, 40, 167-175.

Schlosser, D.A., Zinberg, J.L., Loewy, R.L., Casey-Cannon, S., O’Brien, M.P., Bearden, C.E., ... Cannon, T.D. (2010). Predicting the longitudinal effects of the family environment on prodromal symptoms and functioning in patients at-risk for psychosis. Schizophrenia Research, 118, 69-75.

Subotnik, K.L., Schell, A.M., Chilingar, M.S., Dawson, M.E., Ventura, J., Kelly, K.A., ... Nuechterlein, K.H. (2012). The interaction of electrodermal activity and expressed emotion in predicting symptoms in recentonset schizophrenia. Psychophysiology, 49(8): 1035-1038.

Tarrier, N. y Turpin, G. (1992). Psychosocial factors, arousal and schizophrenic relapse: a review of the psychophysiological data. British Journal of Psychiatry, 161, 3-11.

Tompson, M.C., Goldstein, M.J., Lebell, M.B., Mintz, L.I., Marder, S.R. y Mintz, J. (1995). Schizophrenic patients' perceptions of their relatives' attitudes. Psychiatry Research, 57, 155-167.

Treanor, L., Lobban, F. y Barrowclough, C. (2011). Relatives' responses to psychosis: An exploratory investigation of low expressed emotion relatives. Psychology and Psychotherapy, 86(2), 197-211.

Treanor, L., Lobban, F. y Barrowclough, C. (2013). Relatives' responses to psychosis: An exploratory investigation of low expressed emotion relatives. Psychology and Psychotherapy: Theory, Research and Practice, 86, 197-211.

Treasure, J. y Schmidt, U. (2013). The cognitive-interpersonal maintenance model of anorexia nervosa revisited: a summary of the evidence for cognitive, socio-emotional and interpersonal predisposing and perpetuating factors. Journal of Eating Disorders, 1, 13.

Van Humbeeck, G., Van Audenhove, Ch., De Hert, M., Pieters, G. y Storms, G. (2002). Expressed emotion: a review of assessment instruments. Clinical Psychological Review, 22, 323-343.

Vaughn, C.E. y Leff, J. (1976a). The influence of family and social factors on the course of psychiatric illness. British Journal of Psychiatry, 129, 125-137.

Vaughn, C.E. y Leff, J. (1976b). The measurement of expressed emotion of families of psychiatric patients. British Journal of Social and Clinical Psychology, 15, 157-165. 
Wearden, A.J., Tarrier, N., Barrowclough, C., Zastowny, T.R. y Armstrong, A. (2000). A review of expressed emotion research in health care. Clinical Psychology Review, 20, 5, 633-666.

Weisman, A.G., Rosales, G.A., Kymalainen, J.A. y Armesto, J.C. (2006). Ethnicity, expressed emotion, and schizophrenia patients' perceptions of their family members' criticism. The Journal of Nervous and Mental Diseases, 194, 644-649.

Zubin, J. y Spring, B. (1977). Vulnerability: a new view of schizophrenia. Journal of Abnormal Psychology, 86, 103-126. 